\title{
Comparison of Normal- and Oblique-Incidence Ultrasonic Measurements for the Detection of Environmental Degradation of Adhesive Joints
}

\author{
P. Cawley, A.J. Kinloch and K. Vine \\ Department of Mechanical Engineering \\ Imperial College of Science, Technology and Medicine \\ Exhibition Road, London \\ SW7 2BX, UK
}

\section{Summary}

Normal- and oblique-incidence ultrasonic scans have been conducted on two- and three-layer adhesive-joint specimens exposed to water at $50^{\circ} \mathrm{C}$ for periods of up to 18 months. The joints consisted of aluminium alloy adherend(s) which was subjected to one of four different surface pretreatments prior to being coated (for the two-layer specimens) of bonded (for the three-layer specimens) with an epoxy polymer. Overall, the oblique-incidence technique detected the same types of defect as the normal incidence scans, except when the resolution was too poor to detect the smallest defects. No defect was detected using oblique-incidence scans that could not be detected using normal-incidence scans. In the two-layer specimens, two main types of defects were detected: edge disbonds and micro-defects. Micro-defects were detected in regions remote from the edges and these small-scale, isolated defects appeared took several forms. In the case of the two-layer specimens, the detection of micro-defects was clearly a strong indicator that the interphase of the specimen had been attacked and weakened by the ingressing water molecules and, indeed, the number of such defects correlated to the loss of interfacial toughness. In the case of the three-layer specimens, the extent of edge disbonding was much lower than compared to the corresponding twolayer specimens. Also, no micro-defects were detected ultrasonically in any of the three-layer specimens. However, examination of some of the failure surfaces from the three-layer specimens suggested that some micro-defects may have been present but that they were too small to be detected at the spatial resolution of the ultrasonic scans. Therefore, for the three-layer joint specimens, there were no indicators from the ultrasonic scans which could reveal whether the joint had suffered attack and weakening of the interphase regions by the ingressing moisture. Finally, for all the tests conducted it was very noteworthy that there was no evidence of a gradual change in either the normal- or oblique-incidence reflection coefficient moving into an apparently wellbonded region from either the edge disbonds or the micro-defects, when present. 


\section{Introduction}

Adhesively bonded aluminium-alloy structures are extensively used, particularly in the aerospace industry. Unfortunately, if the adherends are simply degreased or grit blasted prior to bonding, the joints prove to be highly susceptible to environmental attack. This problem is overcome by applying more sophisticated (and expensive) surface pre-treatments [1,2]. A chromic-acid etch (CAE) followed by phosphoric-acid anodisation (PAA) is commonly used in the USA, while a CAE etch followed by chromic-acid anodisation (CAA) is the favoured European aerospace standard. The oxide layer produced by the CAE etch alone is about $0.05 \mu \mathrm{m}$ thick, while those generated by the PAA and CAA treatments when carried out to the relevant standards are about $0.5 \mu \mathrm{m}$ and $3.5 \mu \mathrm{m}$ thick respectively [2]. The oxide layer produced by PAA treatment has a porous honeycomb structure and adhesive (or primer) may flow into the pores during the curing process, so forming a 'micro-composite' interphase layer [1,2]. The extent of this penetration is a function of the pore size and the viscosity of the adhesive used.

The interphase between the bulk adhesive and the bulk adherend formed by the oxide or 'micro-composite' is a major determinant of the susceptibility of the joint to environmental attack. There is therefore a need to monitor the properties of this layer both immediately after a joint is produced and during service. However, this task is very difficult due to the small thickness of the interphase region produced with the standard PAA and CAA processes compared with a typical adhesive layer thickness of about $100 \mu \mathrm{m}$, and an adherend thickness which is generally over 1000 $\mu \mathrm{m}$; and, once the joint has been made, the interphase region is not readily accessible and must be interrogated via the adherend.

There has been considerable interest in this problem for many years and a review of early work in the field is given by Thompson and Thompson [3]. Ultrasonic methods have generally been regarded as the most potentially useful and the bulk of the research effort has been concentrated in this field. Some promising results have been obtained but the capability of the various possible testing techniques has yet to be fully defined. Four basic categories of ultrasonic technique have been investigated [4]. These are ultrasonic reflection coefficient measurements at normal- and/or oblique-incidence [5-8]; Lamb wave measurements [9-11]; the propagation of true guided waves in the adhesive layer [12-15]; and the measurement of the zeroes of the reflection coefficient from the adhesive layer, which is related to the propagation of leaky guided waves along the layer [16-19]. 
In predicting the sensitivity of a particular technique, most researchers have used models that assume (implicitly or explicitly) that changes in the interlayer due to environmental attack are likely to occur relatively uniformly over the area interrogated by the transducer. A spring model has been commonly used in which the adhesive and adherend are connected by normal and tangential spring elements (see, for example, [20]). In a perfect bond, the spring stiffnesses are infinite, corresponding to a rigid or welded interface, and degradation is modelled by a reduction in either or both the normal and tangential stiffnesses. One commonly modelled case is that of a slip interface in which the normal stiffness is unchanged while the tangential stiffness is reduced to zero. This would correspond to a very thin layer of liquid at the interface. Other workers have modelled the interface as a thin solid layer having degraded properties (see, for example, [21]). At realistic products of layer thickness and frequency, this model can be reduced to the spring model with little loss of accuracy.

The realism of models which assume relatively uniform degradation is questionable, given that access to the joint is via the edges, so more severe degradation might be expected in these regions. However, recent work by Moidu et al [22,23] and Lavrentyev and Rokhlin [24] has shown the development of arrays of micro-defects over a wide area and their ultrasonic results were predicted very well by simple spring (or equivalent) models. There is evidence from the work of Moidu et al [22,23] that different adhesive systems may exhibit different behaviour. They tested two adhesive systems, both of which suffered considerable evidence of degradation in destructive mechanical tests. In one case, the degradation was detected ultrasonically and was well represented by a spring model, while in the other case the degradation was not detected by the ultrasonic tests.

If it is assumed that degradation can be represented by spring or equivalent models, it is easy to show that oblique-incidence inspection is likely to be more sensitive than normal-incidence, compression-wave testing. This is clearly the case for a slip interface (i.e. unchanged normal stiffness and tangential stiffness reduced to zero), but also holds for more realistic cases. All the techniques discussed in [4-19] use ultrasonic waves which have components which do not propagate normal to the interface. Calculations based on likely interphase-layer properties [4] indicated that the measurement of oblique-incidence reflection coefficients is likely to be the most promising technique in practice, since its sensitivity to the interlayer characteristics is at least as good as that of the other methods and the reflection coefficients are relatively insensitive to small changes in the bulk adherend and adhesive properties. However, the test involves the accurate 
monitoring of the amplitude of the reflection from an embedded interface which is not a simple task, particularly at oblique incidence.

A previous paper [25] has reported a study of the changes in the ultrasonic reflection characteristics of a series of bonded joints with different pretreatments during exposure to water at $50^{\circ} \mathrm{C}$. The toughness changes have been measured destructively in a double cantilever beam test and have been related to observations of the failure surfaces and the results of periodical normalincidence ultrasonic scans during the degradation. The present paper compares the results obtained using oblique- and normal-incidence ultrasonic measurements which have been conducted as a function of the degradation process of the joints, and discusses the implications for the practical inspection of adhesive joints.

\section{Specimens and Experimental Techniques}

\section{$2.1 \quad$ Specimens}

Two main types of specimen were used, a two-layer and a three-layer specimen, as shown in Fig. 1. Similar two-layer specimens were employed in earlier work by Cawley et al. [8] and also by Wylde and Spelt [26]. The two-layer specimen was chosen for several reasons: an exposed epoxy layer would allow water to diffuse through the epoxy to the interface in a uniform manner, so possibly making the degradation more uniform and easier to detect. Also, when a $2 \mathrm{~mm}$ thick layer is used, the epoxy layer would reach saturation over the course of a few months, and will also give good separation between ultrasonic echoes. The three-layer specimen is more realistic of a typical bonded joint. The adhesive layer will absorb water in a non-uniform manner and, with such a relatively large bonded area, will take several years to reach complete saturation throughout the adhesive layer.

Variations in edge conditions were also investigated, with two of the edges of the specimens being sealed with a marine sealant, and two being left unsealed. Two edges were produced with a flush epoxy/aluminium edge, with the remaining two having an overlap between the edge of the aluminum and the epoxy. The edges with overlap would allow any degradation advancing from the joint edge to be detected immediately, whereas the flush edges could not be inspected ultrasonically to the edge of the epoxy layer, due to the finite size of the probe. Four common pretreatments $[1,2]$ were investigated; grit-blast, chromic-acid etch (CAE), chromic-acid anodising (CAA) and phosphoric acid-anodising (PAA). The aluminium alloy used was a typical aerospace grade (UK L157). 
The three-layer specimen maintained the same geometry as the two-layer specimen, with the exception of the second adherend being present and the thickness of the epoxy layer being reduced to $0.2 \mathrm{~mm}$, which is more realistic of a structural adhesive joint.

Both the two- and three-layer joints were immersed in water at $50^{\circ} \mathrm{C}$ and inspected at regular intervals. The top aluminium surfaces were protected with a rubber sealant while the specimens were immersed. This prevented gross corrosion of the aluminium, so leaving a smooth surface when the sealant was removed prior to ultrasonic testing. (Fresh sealant was re-applied before re-immersing the specimens in the water at $50^{\circ} \mathrm{C}$.) The adhesive used in both specimen types was a two part liquid epoxy supplied by Ciba Geigy, using resin 'AY103' and hardener 'HY951'. The specimens were allowed to cure at room temperature for 48 hours.

\subsection{Ultrasonic Techniques}

Normal- and oblique-incidence 'C-scans' were carried out at regular intervals on all the specimens, after removing them from the water, at $50^{\circ} \mathrm{C}$, immersion tanks. The normal incidence pulse echo scans were performed using a $4 \mathrm{~mm}$ diameter, $25 \mathrm{~mm}$ focal length, $50 \mathrm{MHz}$ focused PVDF transducer manufactured by Krautkrammer. The centre frequency of the received signal was maintained above $50 \mathrm{MHz}$ by using a short water path of 2-3 mm. This produced scans with a spot size of approximately $0.1 \mathrm{~mm}$.

Oblique-incidence inspection is more complicated than normal-incidence, since more waves can propagate. The test configuration usually employed for oblique-incidence inspection is shown schematically in Fig. 2. The joint is immersed in a water bath and a transmitting transducer launches a longitudinal wave through the water at an angle $\theta_{\mathrm{i}}$ to the normal. Reflection, refraction and mode conversion at each interface in the system result in a sequence of echoes reaching a receiving transducer oriented at an angle $-\theta_{\mathrm{i}}$ to the normal. The first echo to be received is that due to the front face reflection from the water-adherend interface. This is followed by a sequence of echoes from the adherend/adhesive interface. When the wave meets the water/adherend interface, some of its energy is reflected and some is transmitted as longitudinal and shear waves in the adherend at angles $\theta_{\mathrm{L}}$ and $\theta_{\mathrm{S}}$ respectively. These transmitted waves then interact with the adherend/adhesive interface. Some of the energy of the longitudinal and shear waves produced by reflection at the interface is then converted into longitudinal waves in the water which are detected by the receiving transducer. 
Four reflection coefficients from the adhesive/adherend interface can be considered: longitudinal wave incident-longitudinal wave reflected (LL), longitudinal-shear (LS), shearlongitudinal (SL) and shear-shear (SS). However, the signals produced by the LS and SL reflections from the interface always appear at the receiver at same time and so cannot be separated. Previous work [8] showed that the shear-shear reflection coefficient at an angle of $32^{0}$ in the aluminium was likely to be the most sensitive to changes in the properties of the oxide layer at the interface. This also has the advantage of being beyond the longitudinal critical angle, so the received signal is simplified. The tests reported in [8] were a series of point measurements, the transducers being aligned carefully at each test point. In the work reported here it was important to scan the specimens so that any localised changes would be detected. It is clearly impractical to re-align the transducers at each point of the scan so the measurement must be relatively insensitive to small changes of angle. Fig. 3 shows the predicted shear-shear reflection coefficient for a bare aluminium/epoxy interface having no oxide layer compared with that for a $3.5 \mu \mathrm{m}$ thick oxide layer at a frequency of $20 \mathrm{MHz}$ assuming the PAA oxide properties measured using acoustic microscopy [27]. The maximum difference between the two cases occurs at about $32^{\circ}$, but this angle is close to the critical angle where the reflection coefficient changes rapidly. Maintaining the shear wave incident angle in a scan to better than one or two degrees is very difficult if the specimen bows slightly during manufacture or exposure. (The accuracy of the transducer-specimen alignment in the water bath has to be greater than that required of the shear wave in the aluminium because refraction increases the change in angle.) It was therefore decided to use a shear wave incident at $37^{\circ}$ in the aluminium adherend, corresponding to $16.8^{\circ}$ in water.

Fig. 4a shows an oblique incidence scan of a three-layer PAA specimen using a pair of 6 mm diameter, $20 \mathrm{MHz}$ unfocussed Panametrics transducers. The features appear blurred and there is an apparent change in the response between the top and bottom of the specimen. It was found that this was due to the specimen being slightly bowed, so that the angle of incidence varied across the specimen. Fig. 4b shows the corresponding scan using a pair of $9 \mathrm{~mm}$ diameter, $10 \mathrm{MHz}$, focused transducers having a focal length in water of $75 \mathrm{~mm}$, the focal region being centred on the interface of interest. The image here is much sharper, indicating that the use of the focused probes reduces the sensitivity of the measurement to misalignment. The use of focused transducers means that the reflection coefficient is effectively measured over a range of angles and Fig. 3 indicates that centring this range on $37^{\circ}$ will give good sensitivity over a significant range of angles on either side of the centre. Having shown that it was essential to use focused transducers for the oblique 
incidence scans, a pair of $20 \mathrm{MHz}, 6 \mathrm{~mm}$ diameter, $50 \mathrm{~mm}$ focal length transducers was obtained. The higher frequency would give better sensitivity to interfacial changes than the $10 \mathrm{MHz}$ transducers of Fig. 4b and it was found that the insensitivity to misalignment was retained. Most of the oblique-incidence work reported here was therefore conducted with the $20 \mathrm{MHz}$, focused transducers.

\subsection{Mechanical Testing}

Mechanical tests were performed on the specimens after they had been immersed in hot water for various lengths of time. A mechanical test that would give spatially discrete information about the toughness of the specimens was desirable. In order to do this, the specimens were cut into $10 \mathrm{~mm}$ wide strips. In the case of the two-layer specimens, each strip was bonded to a stiff base and an end block was glued to one end of the aluminium adherend. A constant rate of displacement was then applied to the end of the specimen, and the load and crack length were measured. Knowing the geometry of the adherend and the load at a given crack length allowed the adhesive fracture energy, $\mathrm{G}_{\mathrm{c}}$, of the bond to be measured [28]. With the specimen bonded to a stiff base the failure tended to propagate at, or very close to the aluminium/epoxy interface [29]. For the three-layer specimens a similar approach was used. In this case end blocks were glued to both aluminium-alloy adherends at one end of the specimen. The failure of these specimens was generally through the epoxy layer, except in the cases where there had been significant degradation of the interface, when the crack would propagate at, or close to, the interface. The measured toughness was then displayed in a similar fashion to a 'C-scan', with a grey-level square representing the toughness, $\mathrm{G}_{\mathrm{c}}$, at a given point on the specimen. The mechanical test results are reported in detail in [25].

\section{Results}

\section{$3.1 \quad$ Two-layer specimens}

Table 1 shows a summary of the results obtained from the two-layer specimens. It was found from the ultrasonic inspections that were periodically conducted that disbonds initiated at the edges of all the two-layer specimens and after initiation their rate of growth depended on the durability imparted by the chosen surface pre-treatment. Isolated micro-defects were seen in regions remote from the edges and again their number and growth depended on the durability imparted by the chosen surface pre-treatment. These two types of defect that were detected are discussed in more detail below.

After the final ultrasonic tests had been completed, the toughness, $G_{c}$, of the specimens was measured as described above [25]. In all cases the locus of joint failure was visually assessed as 
being along the epoxy-adhesive/aluminium-oxide interface, and a similar observation was typically recorded for the unexposed 'control' specimens. It should be noted that the calculation of the average toughness for the degraded sample discounted the regions of the edge disbonds, which had already failed prior to the mechanical tests and so possessed zero toughness. Thus, for all the specimens examined any loss in interfacial toughness is related to the areas which were still bonded, albeit with the presence of some micro-defects. However, the toughness data for the CAA specimens shown in Table 1 reveals an increase for the specimen tested after environmental exposure, compared to the 'control' which was not exposed. This was considered to be due to water being rapidly absorbed by, and plasticising, the epoxy and so increasing the toughness of the epoxy layer. From the results shown in Table 1, the detection of micro-defects is clearly a strong indicator that the interphase of the specimen is being attacked by the ingressing water molecules and, indeed, and number of such defects correlates to the loss of interfacial toughness. Hence, the discussion below concentrates on the detection and interpretation of these micro-defects.

Fig. 5 shows the results obtained from the normal incidence scans of a two-layer grit-blast specimen. The figure label details how many days the sample had been immersed in water at $50^{\circ} \mathrm{C}$ for each of the scan images presented. The scale at the bottom of the figure shows the signal amplitude associated with the grey scale of each image. It is shown as a percentage of the maximum measurable by the digitiser; signal amplitudes above the upper limit are shown in black, and those below the lower limit are shown in white.

The most obvious change to occur with increasing exposure time to water at $50^{\circ} \mathrm{C}$ is the growth of disbonds from the unsealed edges, and these advance rapidly toward the centre of the specimen. From the initial scan there is an area of white speckle which is due to surface roughness. The effect of roughness can also be seen in the reflections from the disbonded regions of the sample; as time progresses, corrosion roughens the surface, and the reflected signal amplitude drops. After 27 days in water there are several changes to be noted. The most important change is disbonding initiating from the unseal ed edges. Outside the bonded area, rapid corrosion of the free unsealed edge can be seen, with large corrosion pits appearing. The marine paint that was used to seal two of the edges can also be seen. This paint was applied after the initial scan but prior to exposure.

However, closer observation shows that there are also some small dark spots appearing under the epoxy. These are apparently quite randomly distributed, with no apparent difference being 
seen with proximity to sealed or unsealed, flush or recessed edges. (Unfortunately many of these small defects are more easily seen on the computer screen than the printed figure.) Some of these small spots have been highlighted on scans taken at longer exposure periods (e.g. Fig. 5c) but close scrutiny of the scan after 27 days shows initial signs of these micro-defects (i.e. relatively small defects which are isolated from adjacent defects and occur throughout the specimen). There are also blurred lighter-shaded lines visible; these are due to scratches on the top surface, produced during removal of the protective rubbery-seal ant coating prior to scanning. As the exposure time increases, the general trend is for many of the features seen after 27 days of exposure to increase in number and to grow in size.

From the present studies, these micro-defects therefore appear to take basically three forms. Firstly, there are small spots which are generally detected when their size is around $0.5 \mathrm{~mm}$ diameter, or bigger. Secondly, there are line defects, which in many cases lead to the development of small corrosion spots. Again their detectability appears to be size limited. Thirdly, there are also darker areas which appear within the central region of the specimen, which visual examination through the transparent epoxy suggested were clusters of extremely small disbonds.

Roughening of the unsealed, recessed edge by corrosion caused the signal to drop below the minimum amplitude threshold on the scan after 67 days in water. (There was still an easily detectable reflection from the interface in this area but it was lower than the minimum that is assigned a grey level in these scans). Therefore, Fig. 6 shows a comparison of a high-resolution normal-incidence scan (0.1 mm spatial sampling) with a photograph of the corresponding area from the two-layer grit-blast specimen shown in Fig. 5. This illustrates that the largest of the microdefects are clearly detected ultrasonically. The scan also shows that defects as small as $0.5 \mathrm{~mm}$ diameter can be detected ultrasonically. The narrow line in the top right of the photograph, which is approximately $0.3 \mathrm{~mm}$ wide is al so apparent from the scan. However the photograph also shows a number of smaller defects that cannot be seen from the scan. These defects are smaller than the resolution that can be obtained using the $50 \mathrm{MHz}$ focused probe.

Fig. 7 shows the oblique-incidence scans corresponding to the normal-incidence scans of Fig. 5. The edge disbonds can clearly be detected, but the smaller micro-def ects are less easily seen and many of them cannot be detected at all. This is due to the lower spatial resolution of the oblique-incidence measurements. Both the oblique and normal-incidence scans show a sharp interface between the disbonds and the surrounding bonded areas, with no evidence of a 
deterioration in the properties of the adhesive layer or the interface adjacent to the disbond being detectable at all.

Fig. 8 shows a comparison of the final normal- and oblique-incidence scans taken for this specimen after 223 days in water, together with a photograph of the specimen looking through the transparent epoxy. A very large number of line and spot micro-defects can be seen in the photograph, some of which are apparent in the normal-incidence scan, a much smaller number being seen on the oblique-incidence scan. It was found that defects as small as $0.5 \mathrm{~mm}$ in diameter could be detected at normal incidence (with some detail being observed at a scal e of $0.1 \mathrm{~mm}$ ), while at oblique incidence the minimum detectable size was around $2 \mathrm{~mm}$. When the failure surfaces were examined in detail [25], it was found that a thin $(<10 \mu \mathrm{m})$ layer of epoxy was left on the surface of the adherend at locations corresponding to some of the micro-defects. Indeed, in the mechanical tests, the main crack was observed to propagate along the weakened interface; except in regions where there was already a micro-defect in the adhesive where the crack deflected through this defect, so leaving a thin layer of adhesive on the adherend surface in this region. Thus, it is suggested that these forms of micro-defects are micro-cracks that have developed in the epoxy, al beit very close to the interface with the adherend, during immersion of the specimen in water at $50^{\circ} \mathrm{C}$. These micro-cracks may act as water paths to allow more ready access of the ingressing moisture, and hence allow more rapid environmental attack on the adjacent interphase regions of the specimen.

Fig. 8 shows scans from a two-layer CAE treated specimen after 194 days in water at $50^{\circ} \mathrm{C}$. Micro-defects remote from the edges are again apparent. These defects can readily be detected given a sufficiently high-resolution scan, as shown in Fig. 9b, but as with the grit-blast samples there were other forms of micro-defects, particularly line defects of the form shown in Fig. 8c, that were visually apparent but were not detectable with ultrasound. Fig. 10 shows the corresponding oblique-incidence scans. Again, the large edge disbonds can readily be seen, but the smaller defects are not evident. It is also interesting to note the pairs of white spots evident on Fig.10a. These correspond to corrosion spots on the top adherend surface where a small amount of corrosion had occurred underneath the rubber sealant that was applied to protect the surface while the specimen was in water. In the oblique-incidence scan, defects at the top surface appear in pairs because a given point on the surface is in the 'line of sight' of the transmitting and receiving transducers at different scan positions. 
Fig. 11 shows a comparison between the normal- and oblique-incidence scans of atwo-layer CAA specimen after 465 days in water at $50^{\circ} \mathrm{C}$, and a photograph taken through the epoxy layer. No micro-defects were seen in this specimen and the failure surface showed no evidence of spots or lines of epoxy such as those that corresponded to micro-defects in the other specimens discussed above. Again, the oblique-incidence scan shows a similar picture to the normal-incidence scan but at an inferior resolution. As before, pairs of white spots corresponding to top-surface corrosion marks can be seen on the oblique-incidence scan and there is no evidence of a gradation of reflection coefficient moving away from the edge of the disbond into the bonded area. The changes in reflectivity that are seen close to the edge of the disbond are inside the disbonded region and are due to corrosion of the surface post-failure; this can also be seen on the photograph. The lack of micro-defects indicates that this surface pretreatment imparts excellent durability to the interphase region of the specimen and this is in complete agreement with the retention of interfacial toughness after water exposure (see Table 1), and the excellent in-service performance that the CAA treatment typically imparts [1,2].

\subsection{Three-layer specimens}

The results from the three-layer specimens are summarised in Table 2. In these specimens the extent of edge disbonding was much lower when compared to the corresponding two-layer specimens. This is undoubtedly due to the addition of the second adherend in the three-layer specimens increasing the stiffness of the system. This prevents the epoxy layer from being forced away from the aluminium surface by the growth of corrosion products on the aluminium surface, and so levering the joint open and accelerating the rate of edge disbonding due to a corrosion mechanism. The grit-blast specimen was exposed for a shorter time period than the other specimens because relatively large edge disbonds became apparent after a relatively short exposure time, whereas there was minimal evidence of edge disbonds on the PAA specimen even after a very long exposure time. No micro-defects were detected ultrasonically in any of the three-layer specimens, but examination of the failure surfaces from the mechanical test of the specimens that failed interfacially (see Table 2) suggested that some defects of this type may have been present. This is discussed further below.

The mechanical tests on the unexposed, 'control', specimens showed that they all failed via cohesive fracture through the adhesive layer. From a visual assessment, the three-layer grit-blast and PAA specimens exposed to water failed interfacially, whilst the failure of the CAE and CAA specimens remained cohesive through the adhesive layer. (Again, the calculation of the average toughness, $G_{c}$, for the degraded samples excluded the regions of any edge disbonding.) However, as 
may be seen from the results shown in Table 1, the exposed specimens that failed cohesively still experienced some loss of toughness. This complex behaviour was found to be due to the deleterious effects of heat on the properties of the epoxy adhesive layer, but without the benefits from the adhesive layer being able to readily absorb moisture and so become plasticised, which would be accompanied by an increase in toughness, as seen in the two-layer specimens. This was verified by exposing specimens to $50^{\circ} \mathrm{C}$ but under low humidity (i.e. 'dry') conditions, and more details of the mechanical test results are discussed elsewhere [25]. However, whilst a full quantitative picture is difficult to discern, clearly the grit-blast and PAA surface pretreatments give joints where environmental attack has occurred in the interphase regions, as evidenced by the change from a cohesive to an interfacial locus of failure after water exposure, and this is accompanied by a significant decrease in the toughness of the joint. The relatively poor performance of the PAA joints is at first sight somewhat surprising, but this has been shown to arise from these joints being prepared without the primer which is typically used in the aerospace standard procedure [30]. The primer was omitted in order to reduce the number of layers in the joint and so to simplify the ultrasonic signals in these tests.

Neither the normal- nor oblique-incidence ultrasonic techniques detected any micro-defects, or any other changes, remote from the edges of any of the three-layer specimens, although both techniques detected disbonds growing in from the edges of the grit-blast and CAE three-layer specimens, see Table 2. Thus, a main conclusion to be drawn is that the micro-defects, which were indicative of environmental attack on the interphase regions and of an accompanying decrease in interface toughness for the two-layer specimens, were not detected in the grit-blast and PAA threelayer specimens, nor indeed in any of the three-layer specimens. Nevertheless, the fracture surfaces of the failed mechanical tests of the grit-blast and PAA specimens showed fine lines of epoxy; i.e. showed sign of micro-defects. These surface features were similar to those which corresponded to the micro-defects which were detectable by the ultrasound techniques in the two-layer specimens; although they were smaller in number and in size on the failure surfaces of the grit-blast and PAA three-layer specimens. Therefore, it is possible that micro-defects remote from the edges were present in the three-layer specimens that failed interfacially and suffered a decrease in interfacial toughness, but clearly they were not able to be detected ultrasonically.

This possibility is further supported from the visual observations on the two-layer specimens which clearly revealed that many more micro-defects were present than were detected ultrasonically, only the wider lines or larger spots being visible with ultrasound. (The two opaque 
adherends of the three-layer specimens meant, of course, that visual inspection of the interface was not possible with the three-layer specimens. Hence, for the three-layer specimens, only the appearance of the failure surfaces of the specimens which fractured interfacially can assist on this point.) There are two possible reasons for the smaller number and size of micro-defects in the gritblast and PAA three-layer specimens. Firstly, it is possible that the extra stiffness of the three-layer samples restricted the growth of the micro-defects to below that required for ultrasonic detection. Secondly, since the concentration of water in the adhesive layer at any time would be far lower in the three-layer specimens than the two-layer specimens, it may be that this factor that limited the initiation and subsequent growth of the micro-defects in the three-layer specimens to below that required for ultrasonic detection.

\section{Discussion and Conclusions}

Normal- and oblique-incidence ultrasonic scans have been conducted on two and three-layer adhesive joint specimens exposed to water at $50^{\circ} \mathrm{C}$ for periods of up to 18 months. It was possible to maintain a centre frequency of around $50 \mathrm{MHz}$ in the normal-incidence inspection when testing through an aluminium-alloy adherend thickness of $4 \mathrm{~mm}$. The focused transducer used gave a spot size of about $0.1 \mathrm{~mm}$. Oblique-incidence scanning with unfocused transducers, so obtaining the reflection coefficient at a precise angle of incidence, was not feasible because of the difficulty of maintaining alignment across a specimen which could be slightly bowed. However, satisfactory results monitoring the shear-shear reflection coefficient from the adhesive/adherend interface were obtained using a pair of $20 \mathrm{MHz}$ focused transducers. Increasing the frequency to much above 20 $\mathrm{MHz}$ would probably not be feasible because the attenuation of shear waves in the aluminium would increase and alignment problems would return. Overall, the oblique-incidence technique detected the same types of defect as the normal incidence scans, except when the resolution was too poor to detect the smallest defects. No defect was detected using oblique-incidence scans that could not be detected using normal-incidence scans.

In the two-layer specimens, two main types of defects were detected: edge disbonds and micro-defects. Edge disbonds were simple to detect using both techniques. However, the smaller footprint on the surface of the single, normal-incidence transducer compared to the pair of transducers required for oblique-incidence measurements meant that it was possible to inspect closer to the edges using normal incidence. This would be important for the inspection of loaded joints, since the edges are typically regions of maximum stress and where the water first enters the joint, so it will be important to find small disbonds in these regions. 
Micro-defects were seen in regions remote from the edges and these small-scale, isolated defects appeared to take basically three forms. Firstly, there were small spots which were generally detected when their size was around $0.5 \mathrm{~mm}$ diameter or bigger. Secondly, there were line defects, which in many cases led to the development of small corrosion spots. Again their detectability appeared to be size limited. Thirdly, there were also darker areas which appear within the central region of the specimen, which visual examination through the transparent epoxy suggested were clusters of extremely small disbonds. Many of these micro-defects could be seen visually through the transparent epoxy adhesive which coated the two-layer specimen and some, but not all, could be detected in the normal-incidence ultrasonic scans. However, only the largest of these micro-defects could be seen in the oblique-incidence scans due to the poorer spatial resolution. Finally, it is very noteworthy, that there was no evidence of a gradual change in either the normal- or obliqueincidence reflection coefficient moving into an apparently well-bonded region from either the edge disbonds or the micro-defects. In the case of the two-layer specimens, the detection of microdefects was clearly a strong indicator that the interphase of the specimen had been attacked and weakened by the ingressing water molecules and, indeed, the number of such defects correlated to the loss of interfacial toughness.

In the case of the three-layer specimens, the extent of edge disbonding was much lower than compared to the corresponding two-layer specimens. This was undoubtedly due to the addition of the second adherend in the three-layer specimens increasing the stiffness of the system. This prevented the epoxy layer from being forced away from the aluminium surface by the growth of corrosion products on the aluminium surface, and so levering the joint open and accelerating the rate of edge disbonding due to a corrosion mechanism. However, no edge disbonding was observed on the PAA specimen, although this joint was attacked and significantly weakened by the ingressing moisture. Another major finding was that no micro-defects were detected ultrasonically in any of the three-layer specimens. Although examination of the failure surfaces from the mechanical tests of the grit-blasted and PAA three-layer specimens, that failed interfacially, suggested that some micro-defects may have been present. However, if this was the case, then clearly they were too small to be detected at the spatial resolution of the ultrasonic scans. (It would be difficult to improve the spatial resolution by increasing the frequency of the normal-incidence inspection because the attenuation in the adherend would increase rapidly.) Therefore, it is possible that micro-defects remote from the edges were present in the grit-blast and the PAA three-layer specimens that failed interfacially and suffered a decrease in interfacial toughness, but that they 
were not able to be detected ultrasonically. Finally, again, there was no evidence of a gradual change in either the normal- or oblique-incidence reflection coefficient moving into an apparently well-bonded region from the edge disbonds, when present.

These tests therefore suggest that the most practical way to detect environmental degradation in aluminium/adhesive-layer/aluminium adhesive joints is to look for disbonds at the edges and micro-defects throughout the joint. This is best done using high-frequency, normal-

incidence, focused ultrasound. The frequency and focal length may be optimised to obtain the smallest possible spot size at the adhesive/adherend interface given the thickness of the adherends.

\section{Acknowledgements}

The authors are grateful to support of the work by DERA (UK). 


\section{References}

1. Kinloch, A.J. (1983). 'Durability of Structural Adhesives.' Applied Science, London and New York.

2. Davies, R.J. and Kinloch, A.J. (1989). 'The surface characterisation and adhesive bonding of aluminium.’ in Adhesion 13, K.W. Allen (ed.), Elsevier, London, pp. 8-22.

3. Thompson, R.B. and Thompson, D.O. (1991). 'Past experiences in the development of tests for adhesive bond strength.' J. Adhesion Sci. Technol., 5: 583-599.

4. Cawley, P., Pialucha, T.P. and Lowe, M.J.S. (1993). 'A comparison of different methods for the detection of a weak adhesive/adherend interface in bonded joints.' Review of Progress in Quantitative NDE, 12, D.O. Thompson and D.E. Chimenti (eds.), Plenum Press, New York, pp.1531-1538.

5. Rokhlin, S. I. and Marom, D. (1986). 'Study of adhesive bonds using low-frequency obliquely incident ultrasonic waves.’ J. Acoust. Soc. Am., 80: 585-590.

6. Pilarski, A. and Rose, J.L. (1988). 'Ultrasonic oblique incidence for improved sensitivity in interface weakness determination.’ NDT International, 21: 241-246.

7. Jiao, D. and Rose, J.L. (1991). 'An ultrasonic interface layer model for bond evaluation.' J. Adhesion Sci. Technol., 5: 631-646.

8. Cawley, P., Pialucha, T.P. and Zeller, B.D. (1996). 'The characterisation of oxide layers in adhesive joints using ultrasonic reflection measurements.' Proc. Royal Soc. Lond., Series A, 452:1903-1926.

9. Rokhlin, S. I. and Wang, Y.J. (1991). 'Analysis of boundary conditions for elastic wave interaction with an interface between two solids.’ J. Acoust. Soc. Am., 89:

10. Bar-Cohen, Y. and Mal, A.K. (1990). in Review of Progress in Quantitative NDE, Vol. 9B, p.1271 (1990).

11. Jungman, A., P. Guy, P., Nayfeh, A. and Quentin, G. (1991). in Review of Progress in Quantitative NDE, Vol. 10B, p.1319.

12. Claus, R.O. and Kline, R.A. (1979). 'Adhesive bondline interrogation using Stoneley wave methods.' J. Applied Physics, 60: 8066-8069.

13. Rokhlin, S. and Hefets, M. et al. (1981). 'An ultrasonic interface-wave method for predicting the strength of adhesive bonds.' J. Applied Physics, 52: 2847-2851.

14. Pilarski, A. (1985). 'Ultrasonic evaluation of the adhesion degree in layered joints.' Materials Evaluation, 43: 765-770.

15. Nagy, P.B. and Adler, L., in Review of Progress in Quantitative NDE, Vol. 10B, p.1295 (1991). 
16. Pilarski, A., and Rose J.L., et al. (1990). 'The angular and frequency characteristics of reflectivity from a solid layer embedded between two solids with imperfect boundary conditions.' J. Acoust. Soc. Am., 87: 532-542.

17. Wang, W. and Rokhlin, S.I. (1991). 'Evaluation of interfacial properties in adhesive joints of aluminium alloys using angle-beam ultrasonic spectroscopy', J. Adhesion Sci. Technol., 5: 647-666.

18. Li, B., Hefetz, M. and Rokhlin, S.I. (1992). in Review of Progress in Quantitative NDE, Vol. $\underline{11 B}$, p.1221.

19. Lavrentyev, A.I. and Rokhlin, S.I. (1994). 'Ultrasonic evaluation of environmental degradation of adhesive joints', Review of Progress in Quantitative NDE, 13, D.O. Thompson and D.E. Chimenti (eds.), Plenum Press, New York, pp.1539-1546.

20. Tattersall, H.G. (1973). 'The ultrasonic pulse-echo technique as applied to adhesion.' J. Applied Physics D: Applied Physics, 6: 819-832.

21. Lavrentyev, A.I. and Rokhlin, S.I.(1994). 'Models for ultrasonic characterisation of environmental interfacial degradation in adhesive joints.' in Review of Progress in Quantitative NDE. 1994: Plenum Press, New York.

22. Moidu, A.K., Sinclair, A.N. and Spelt, J.K. (1998). 'Adhesive joint durability assessed using open-faced peel specimens.' J. Adhesion, 65: 239-257.

23. Moidu, A.K., Sinclair, A.N. and Spelt, J.K. (1999). 'Nondestructive characterisation of adhesive joint durability using ultrasonic reflection measurements', Research in Nondestructive Evaluation, 11: 81-95.

24. Lavrentyev, A.I. and Rokhlin, S.I. (1994). 'Models for ultrasonic characterisation of environmental degradation of interfaces in adhesive joints.' J Applied Physics, 76: 4643-4650.

25. Cawley, P., Kinloch, A.J. and Vine, K., 'Correlation of Non Destructive Measurements and Toughness Changes in Adhesive Joints During Environmental Attacks', in preparation.

26. Wylde, J.W. and Spelt, J.K. (1998). 'Measurement of adhesive joint fracture properties as a function of environmental degradation.' Int. J. Adhesion and Adhesives, 18: 237-24.

27. Zinin, P., Lefeuvre, O., Briggs, G.A.D., Zeller, B.D., Cawley, P., Kinloch, A.J., Zhou, X. and Thompson, G.E. (1999). 'Determination of density and elastic constants of a thin PAA oxide film by acoustic microscopy.' J. Acoust. Soc. Am., 106: 2570-2567.

28. B. Blackman, Dear, J.P., Kinloch, A.J. and Osiyemi, S. (1991). 'The calculation of adhesive strain energies from double-cantilever beam test specimens.' J. Materials Science Letters, 10: 253-256.

29. Duer, R., Katevatis, D., Kinloch, A.J. and Williams, J.G. (1995). 'Comments on mixed-mode fracture in adhesive joints.' Int. J. of Fracture, 75:157-162. 
30. Kinloch, A.J., Little, M.S.G. and Watts, J.F. (2000). 'The role of the interphase in the environmental failure of adhesive joints.' Acta Materilia, 48: 4543-4553. 


\section{Figure Captions}

Figure 1 Two- and three-layer specimen design.

Figure 2 Schematic diagram of reflections from an imbedded interface.

Figure 3 Variation in reflection coefficient with angle for an aluminium/epoxy interface with and without an oxide interlayer at $20 \mathrm{MHz}$.

Figure 4 Comparison of $20 \mathrm{MHz}$ oblique-incidence scan from a three-layer PAA specimen (a) using unfocused and (b) focused transducers.

Figure 5 Normal-incidence scans from two-layer grit-blast specimens after exposure for (a) 0 days (b) 27 days (c) 67 days (d) 117 days exposed to water at $50^{\circ} \mathrm{C}$.

Figure 6 Comparison of (a) high-resolution normal-incidence scan and (b) photograph of a corresponding region from the two-layer grit-blast specimen, after 67 days exposed to water at $50^{\circ} \mathrm{C}$.

Figure 7 Oblique-incidence scans from the two-layer grit-blast specimen after exposure for (a) 0 days (b) 27 days (c) 67 days and (d) 117 days exposed to water at $50^{\circ} \mathrm{C}$.

Figure 8 Comparison of (a) normal- and (b) oblique-incidence scan with (c) a photograph from a two-layer grit-blast specimen after 223 days in water at $50^{\circ} \mathrm{C}$.

Figure 9 Normal-incidence scan from two-layer CAE specimen after 194 days exposed to water at $50^{\circ} \mathrm{C}$, with high resolution scan of the line micro-defect.

Figure 10 Oblique-incidence scans from a two-layer CAE specimen after 194 days exposed to water at $50^{\circ} \mathrm{C}$ showing (a) the whole specimen and (b) a high-resolution scan of the highlighted region.

Figure 11 Comparison of (a) normal- and (b) oblique-incidence scans with (c) photograph of the two-layer CAA specimen after 465 days in water at $50^{\circ} \mathrm{C}$. 
Table 1 . Summary of results from the two-layer specimens.

\begin{tabular}{|l|l|l|l|}
\hline Specimen & $\begin{array}{l}\text { Edge disbond rate } \\
\left(\mathbf{m m}^{2} / \mathbf{d a y}\right)\end{array}$ & Micro-defects & $\begin{array}{l}\text { Average toughness } \\
\text { retained (\%) }\end{array}$ \\
\hline Grit blast & 4.8 & Many & 46 \\
\hline CAE & 3.7 & V. few & 96 \\
\hline PAA & 3.2 & Few & 55 \\
\hline CAA & 2.2 & None & 126 \\
\hline
\end{tabular}

Table 2. Summary of results from the three-layer specimens.

\begin{tabular}{|l|l|l|l|l|l|}
\hline Specimen & $\begin{array}{l}\text { Total exposure } \\
\text { time (days) }\end{array}$ & $\begin{array}{l}\text { Disbond Area } \\
\left.\mathbf{( m m}^{\mathbf{2}}\right)\end{array}$ & $\begin{array}{l}\text { “'Wet” locus } \\
\text { of failure }\end{array}$ & $\begin{array}{l}\mathbf{G}_{\mathbf{C}} \text { (wet) } \\
\mathbf{J m}^{-\mathbf{2}}\end{array}$ & $\begin{array}{l}\text { Toughness } \\
\text { retained (\%) }\end{array}$ \\
\hline Grit blast & 251 & 25 & Interfacial & $48( \pm 16)$ & 37 \\
\hline CAE & 411 & 300 & Cohesive & $84( \pm 19)$ & 65 \\
\hline PAA & 566 & $\sim 0$ & Interfacial & $55( \pm 25)$ & 42 \\
\hline CAA & 566 & $\sim 0$ & Cohesive & $95( \pm 28)$ & 73 \\
\hline
\end{tabular}


2-layer specimen

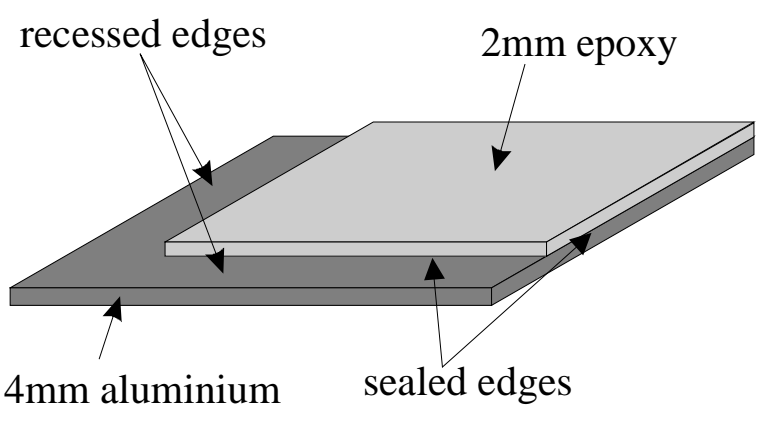

3-layer specimen

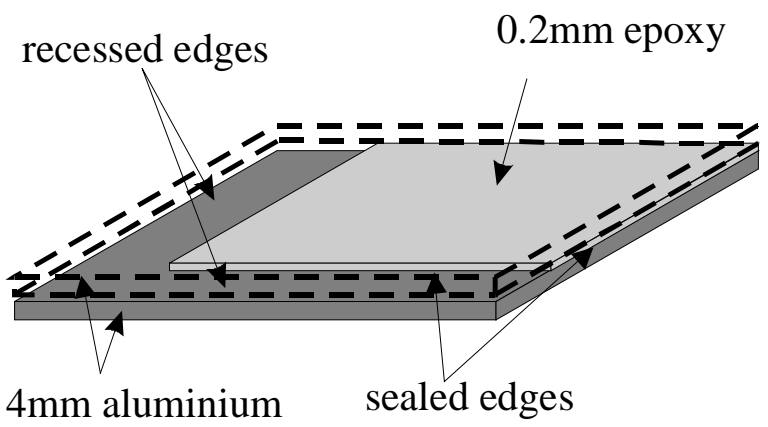

Figure 12 Two- and three-layer specimen design.

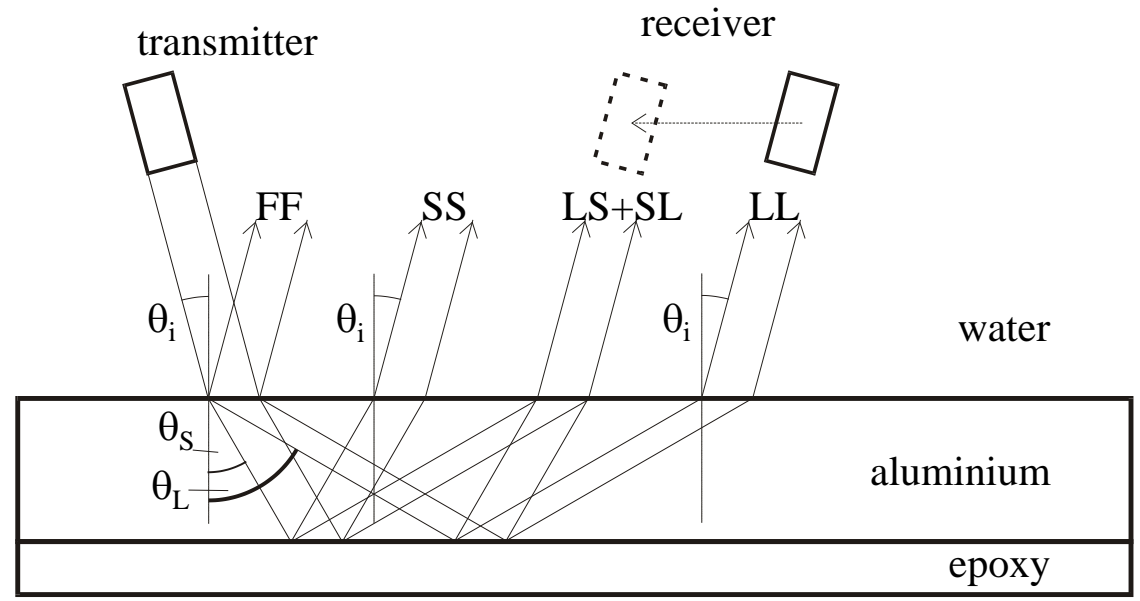

Figure 13 Schematic diagram of reflections from an imbedded interface. 


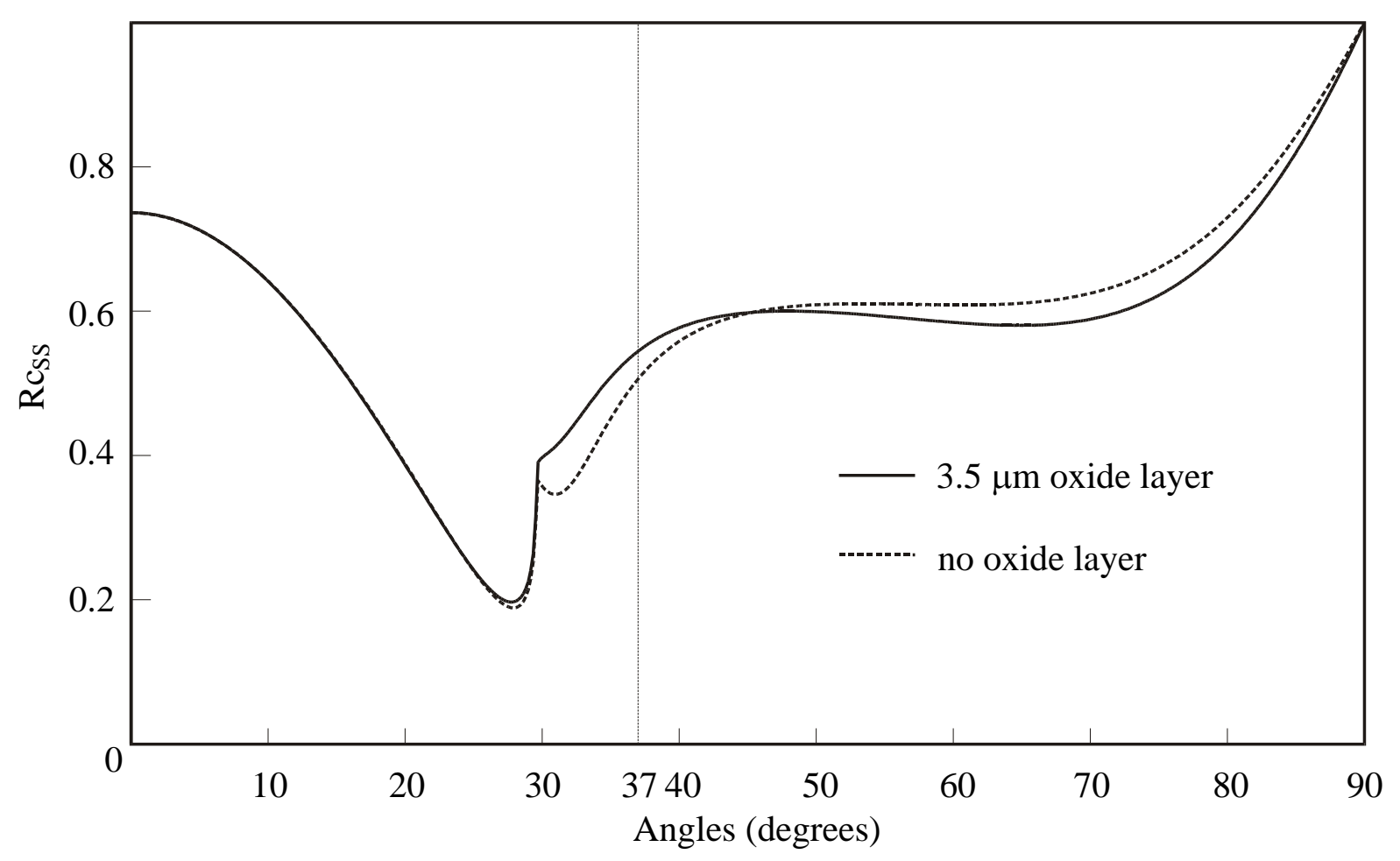

Figure 14 Variation in reflection coefficient with angle for an aluminium/epoxy interface with and without an oxide interlayer at $20 \mathrm{MHz}$.

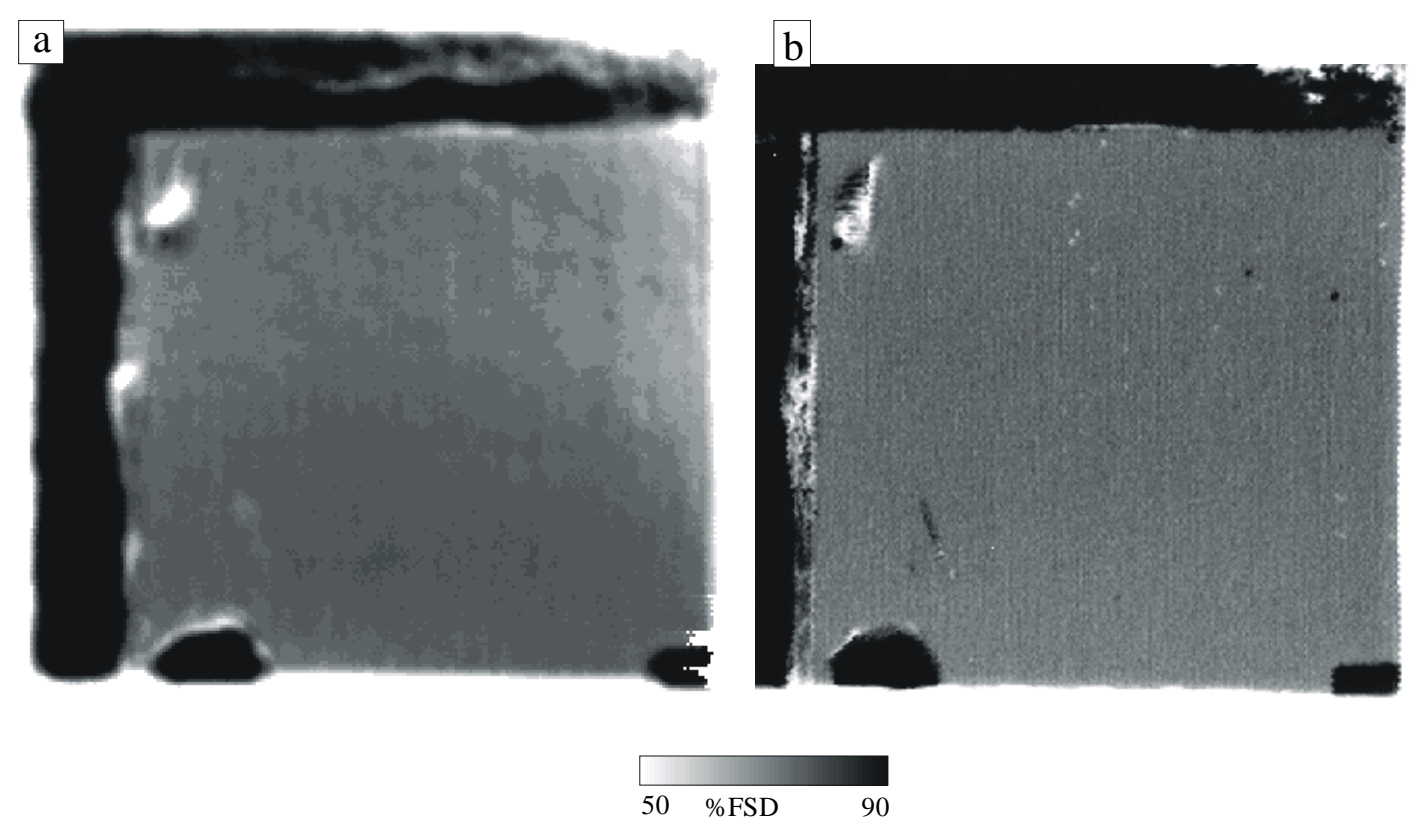

Figure 15 Comparison of $20 \mathrm{MHz}$ oblique-incidence scan from a three-layer PAA specimen (a) using unfocused and (b) focused transducers. 

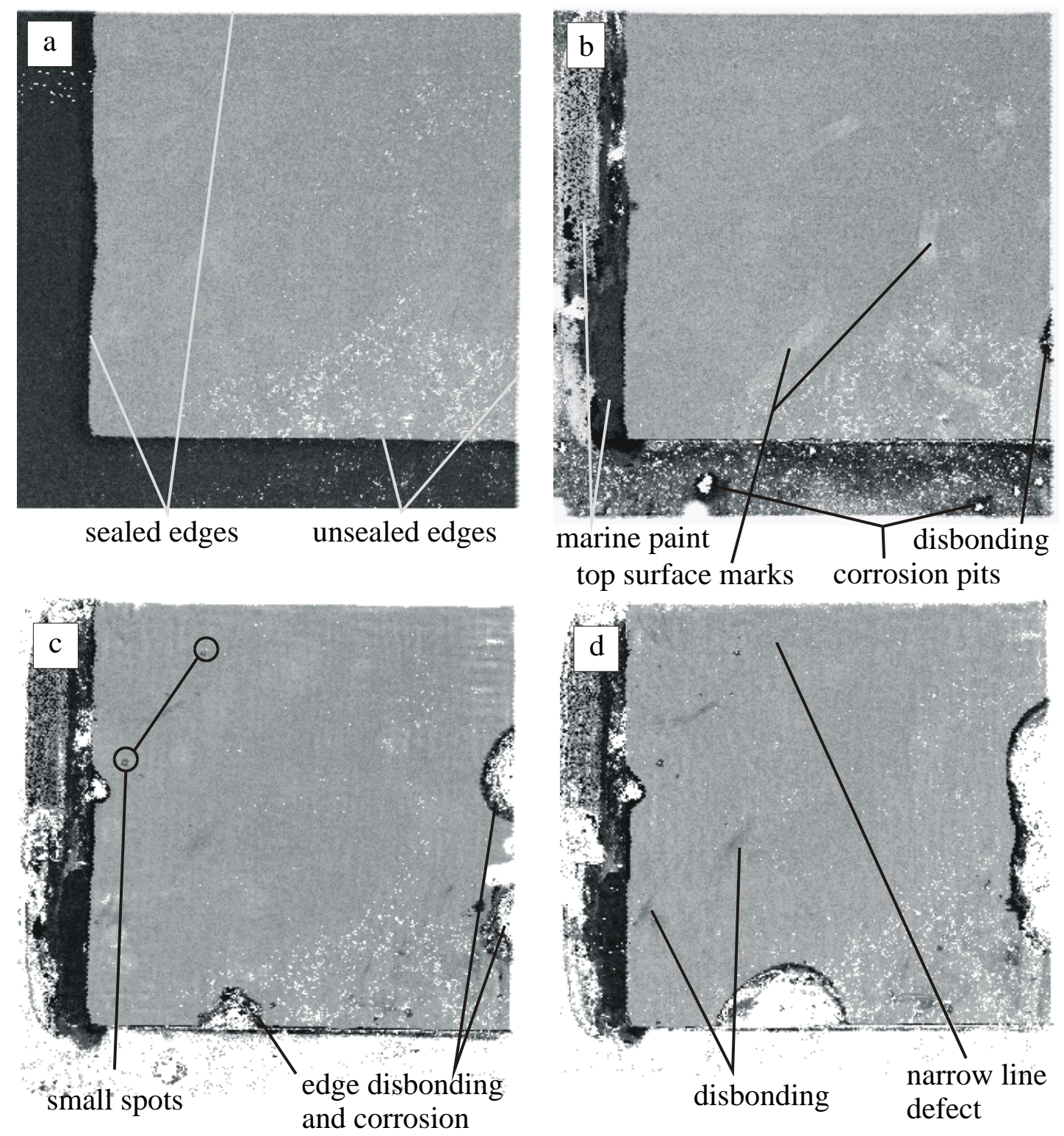

Figure 16 Normal-incidence scans from two-layer grit-blast specimens after exposure for (a) 0 days (b) 27 days (c) 67 days (d) 117 days exposed to water at $50^{\circ} \mathrm{C}$. 

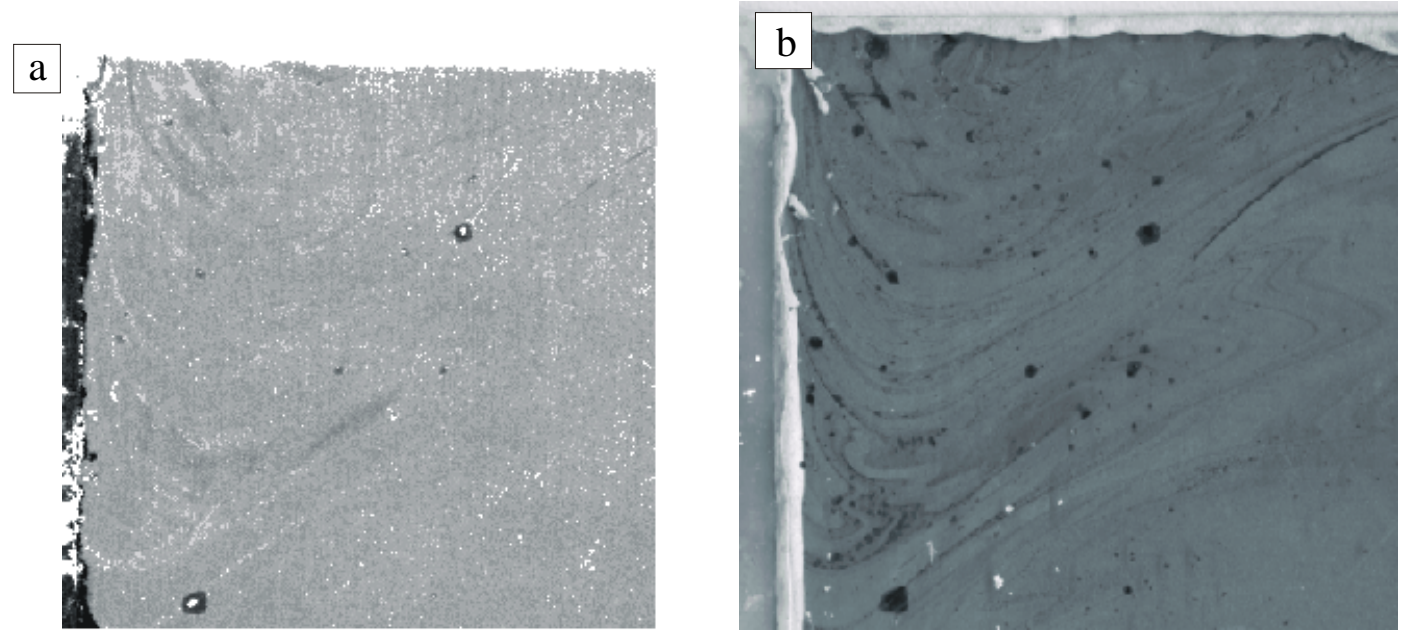

Figure 17 Comparison of (a) high-resolution normal-incidence scan and (b) photograph of a corresponding region from the two-layer grit-blast specimen, after 67 days exposed to water at $50^{\circ} \mathrm{C}$. 


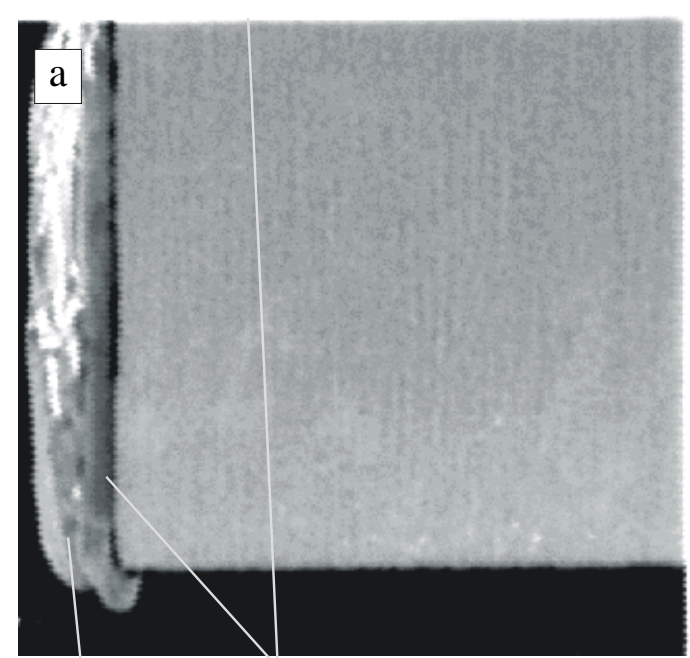

marine paint

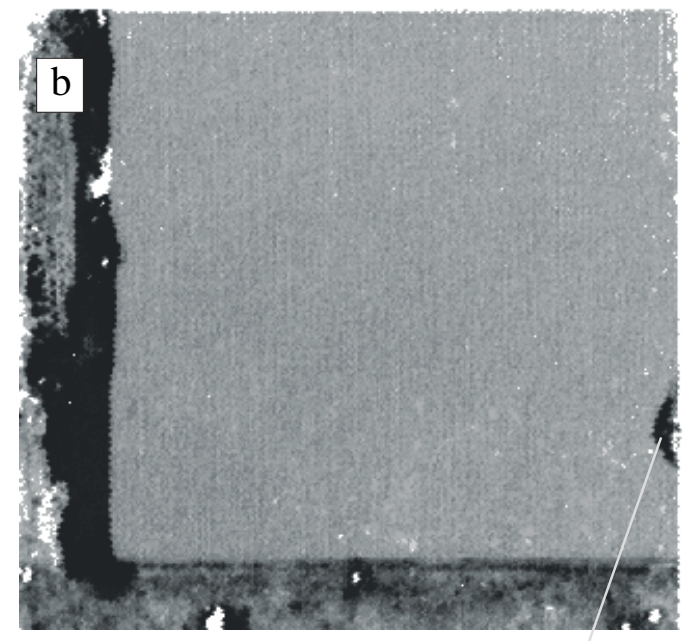

disbond

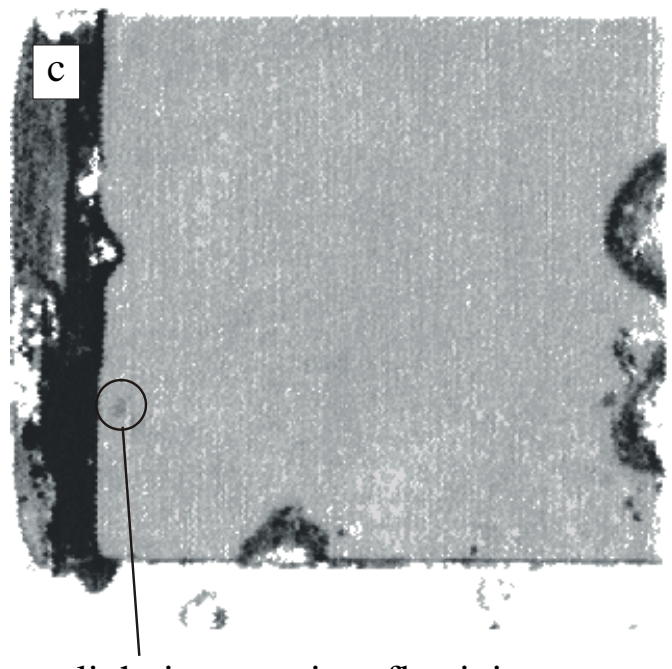

slight increase in reflectivity

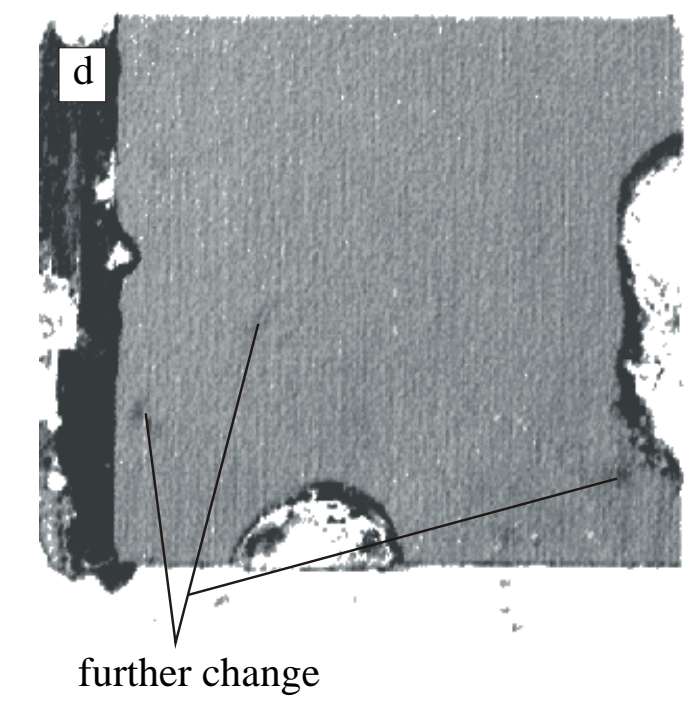

Figure 18 Oblique-incidence scans from the two-layer grit-blast specimen after exposure for (a) 0 days (b) 27 days (c) 67 days and (d) 117 days exposed to water at $50^{\circ} \mathrm{C}$. 
(a)

Line defects detectable

Larger of micro defects detected

Clusters of small defects merge to form general increase in reflectivity

Corrosion driven disbonds from edges easily detected.

Surface roughening due to corrosion behind crack front reduces signal

General areas of high defect density detected but individual defects not identifiable in these areas.

Large corrosion driven edge disbonds clearly detected

(c)

Huge number of defects visible under the epoxy
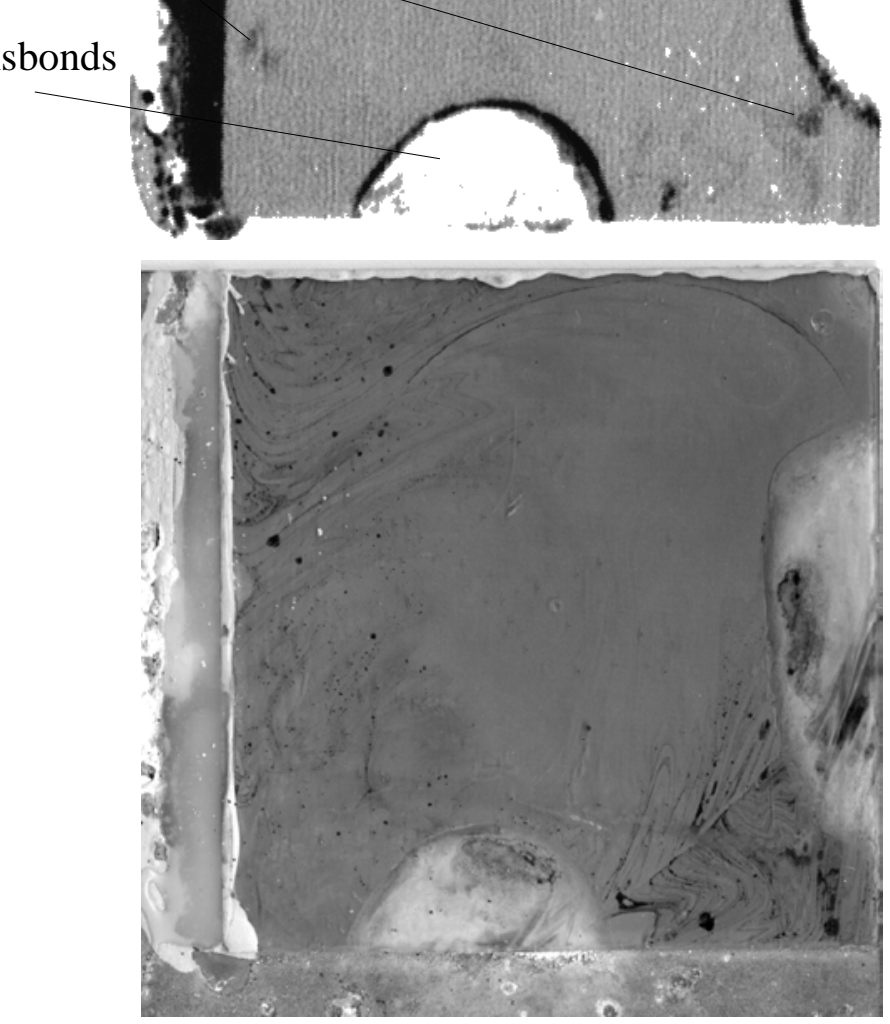

Figure 19 Comparison of (a) normal- and (b) oblique-incidence scan with (c) a photograph from a two-layer grit-blast specimen after 223 days in water at $50^{\circ} \mathrm{C}$. 


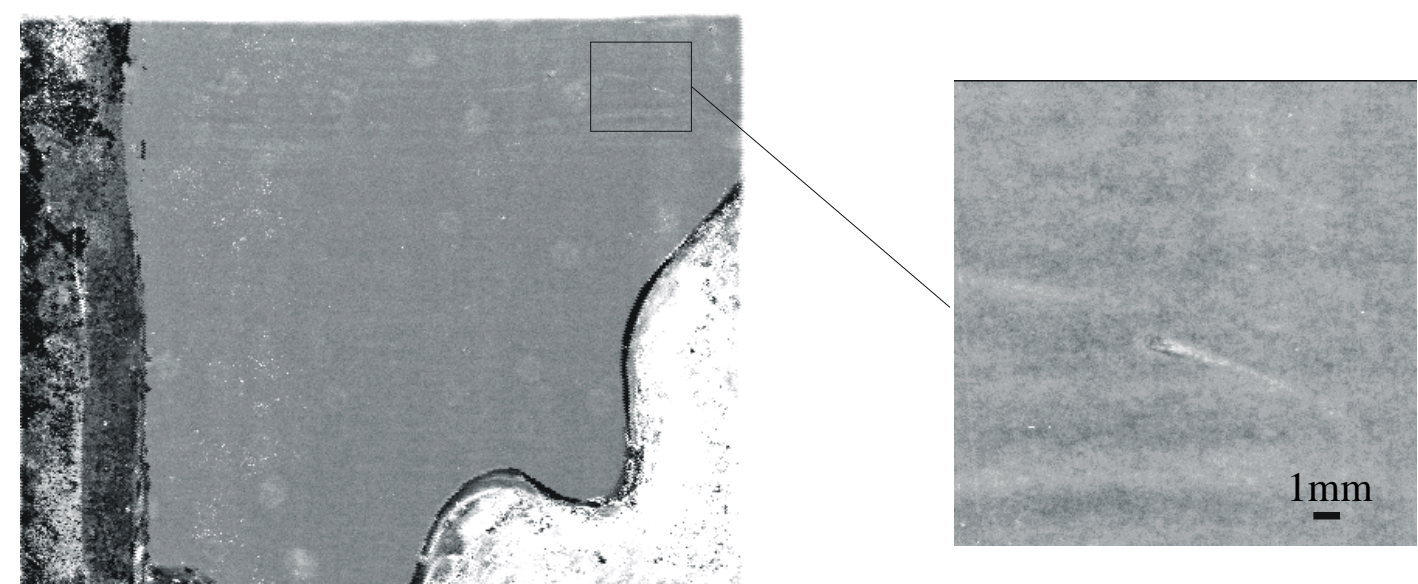

high resolution scan of line defect

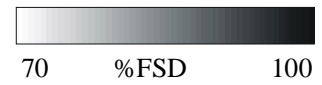

Figure 20 Normal-incidence scan from two-layer CAE specimen after 194 days exposed to water at $50^{\circ} \mathrm{C}$, with high resolution scan of the line micro-defect.
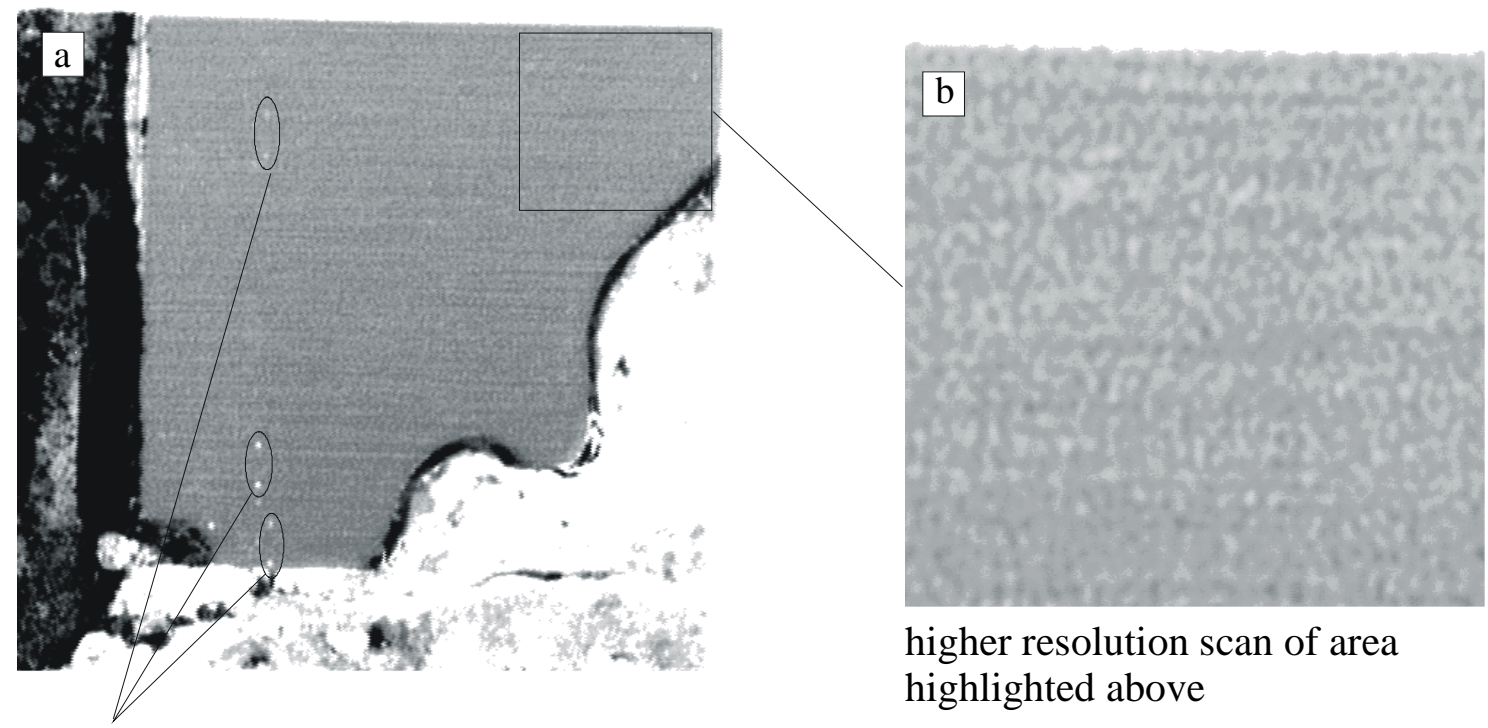

pairs of marks from top surface pitting

Figure 21 Oblique-incidence scans from a two-layer CAE specimen after 194 days exposed to water at $50^{\circ} \mathrm{C}$ showing (a) the whole specimen and (b) a high-resolution scan of the highlighted region. 
(a)

Some top surface corrosion spots interfere with scan

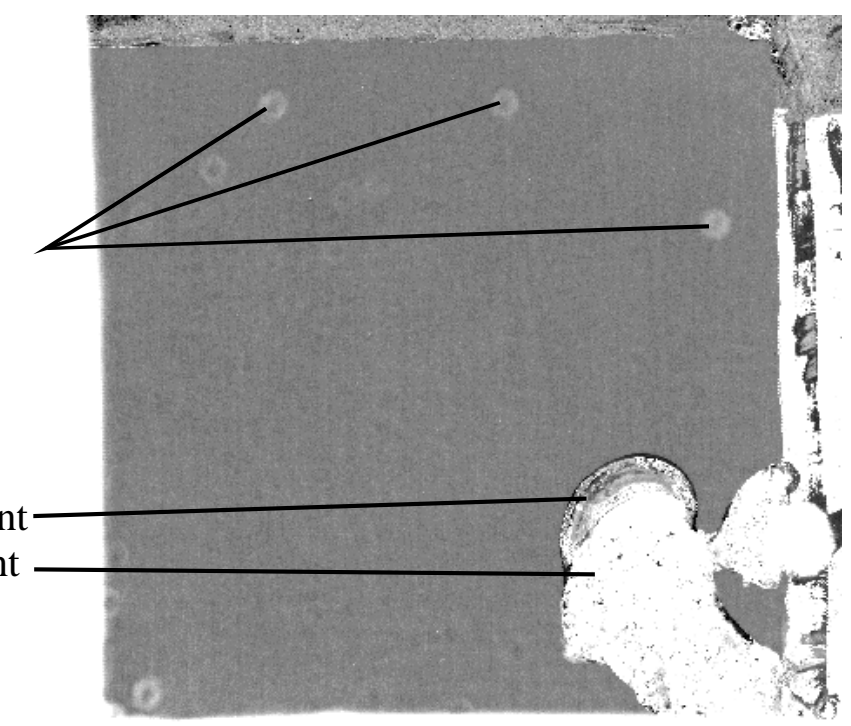

(b)

Top surface corrosion pits leave double shadows on interface scan

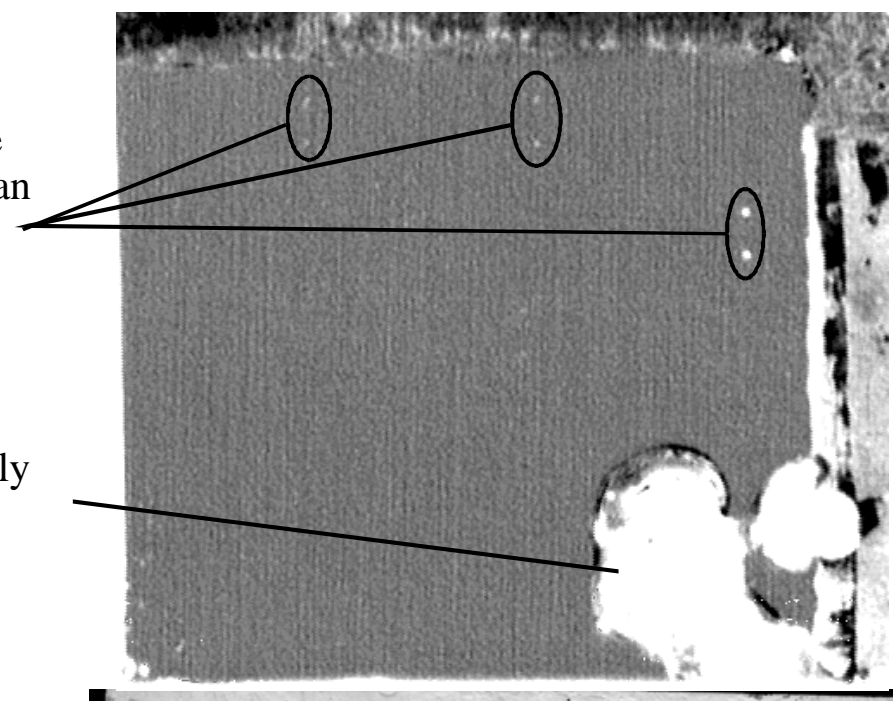

(c)

Large corrosion induced disbond detectable, difference between recent disbond and corroded areas apparent

\section{cAAs}

Corrosion induced disbond easily detected

Free epoxy surface marks, not at interface

Cracks through thickness of the epoxy layer

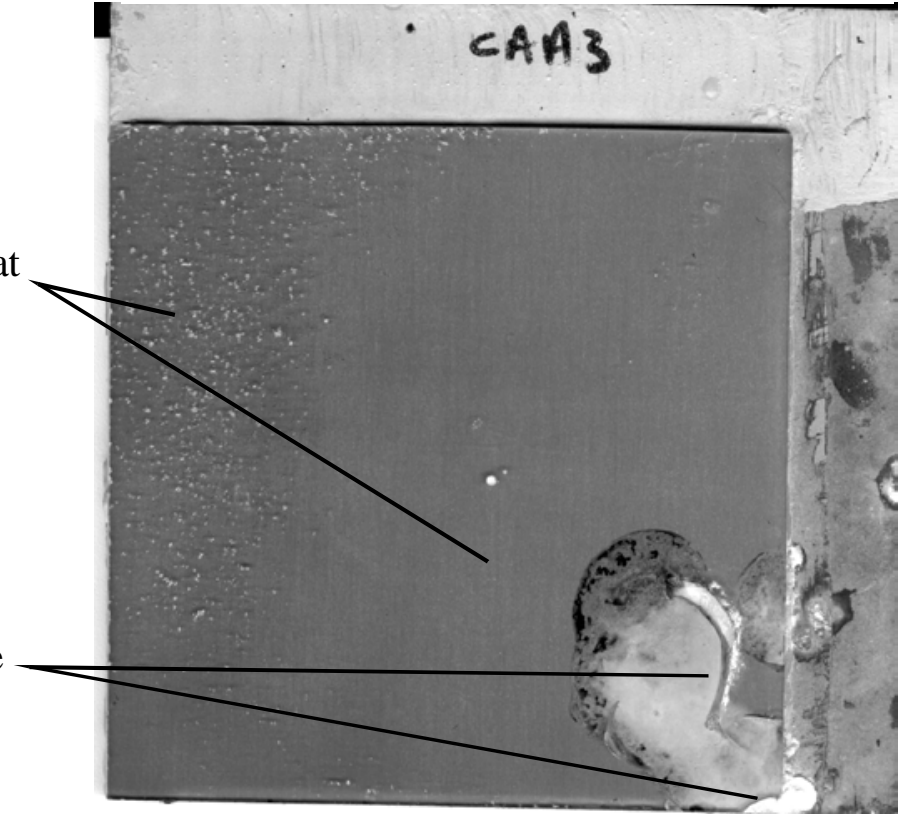

Figure 22 Comparison of (a) normal- and (b) oblique-incidence scans with (c) photograph of the two-layer CAA specimen after 465 days in water at $50^{\circ} \mathrm{C}$. 